\title{
CIRCULATING VASCULAR ENDOTHELIAL GROWTH FACTOR INDEPENDENTLY ASSOCIATES WITH WORSE POST-STROKE OUTCOME
}

Authors: Olof Anger ${ }^{1}$, Katarina Jood ${ }^{2}$, Christian Blomstrand ${ }^{2}$, Ulf Andreasson ${ }^{3}$, Kaj Blennow ${ }^{3}$ Henrik Zetterberg ${ }^{3}$, Jorgen Isgaard', Christina Jern ${ }^{2,4}$, Johan Svensson ${ }^{1}$ N. David Aberg $^{1,5}$ 1 Institute of Medicine, The Sahlgrenska Academy at University of Gothenburg, ${ }^{2}$ Department of Clinical Neuroscience, Institute of Neuroscience and Physiology, The Sahlgrenska Academy at University of Gothenburg, ${ }^{3}$ Department of Neurochemistry, House V3, Institute of Laboratory Medicine Chinical Chemistry, SE-431 80 Mölndal, The Sahlgrenska Academy at University of Gothenburg ${ }^{4}$ Department of Clinical Genetics, The Sahlgrenska Academy at University of Gothenburg ${ }^{5}$ Center of Brain Repair
Academy at University of Gothenburg, Gothenburg, Sweden. \section{(1)}

\author{
Depart
} Correspondence: Dr Olof Anger of Internal Medicine, Sahlgrenska University
Hospital, University of Gothenburg, Gröna Stråket 8, SE-413 45 Göteborg, Sweden. Phone: +46 739 109778, E-mail: olof.anger@gu.se

\section{HIGH SERUM VASCULAR ENDOTHELIAL GROWTH FACTOR IS MODERATELY ASSOCIATED WITH POOR LONG-TERM OUTCOME, WHICH IS NOT MEDIATED BY INITIAL STROKE SEVERITY}

\section{Background and Aims:}

Vascular endothelial growth factor (VEGF) is involved in angiogenesis, lymphogenesis, neuroprotection and brain plasticity. VEGF is found both locally in the brain and in the more easily accessible serum. In some studies, serum-VEGF (s-VEGF) correlates with worse stroke severity. However, most studies on s-VEGF and outcome have shown variable results, have lacked long term follow-up, or have been limited by small study populations. Our aim was to study if s-VEGF at the acute stage and after three months was associated with ischemic stroke (IS) severity and long-term functional outcome.

\section{Method:}

S-VEGF was analyzed in patients $(n=492,36 \%$ females, mean age 57 years) included from the Sahlgrenska Academy Study on Ischemic Stroke (SAHLSIS). Stroke severity was classified according to the National Institutes of Health Stroke Scale (NIHSS), divided into quintiles and 2-year outcome was evaluated using the modified Rankin Scale (mRS).

\section{Results:}

Acute crude s-VEGF did not correlate with crude NIHSS at baseline $(p=0.227 n=489)$ and there was no significant effect of NIHSS-quintile on acute s-VEGF (Fig.1). High s-VEGFquintiles in the acute phase were associated with 2-year $\mathrm{mRS}$ 3-6 adjusted for sex and age (ptrend=0.032). After adding traditional cardiovascular risk factors, the association was weakened ( $p$ trend=0.06) but with additional adjustment for initial stroke severity significance was retained (ptrend=0.046).

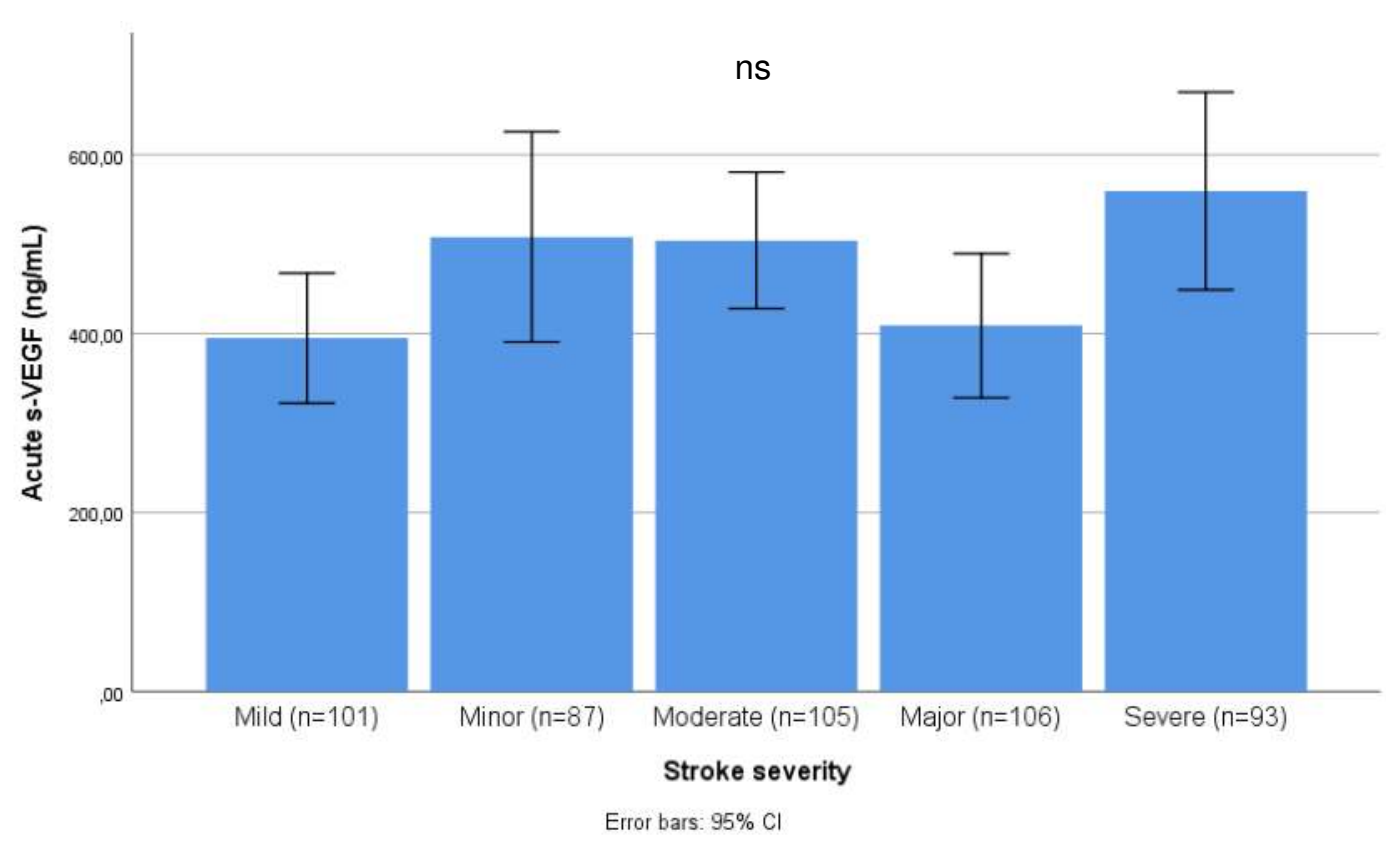

Figure 1. Acute phase s-VEGF in relation to IS severity (quintiles). Significance levels were analyzed by ANOVA followed by post-hoc Dunnett's test with the first quintile as reference.

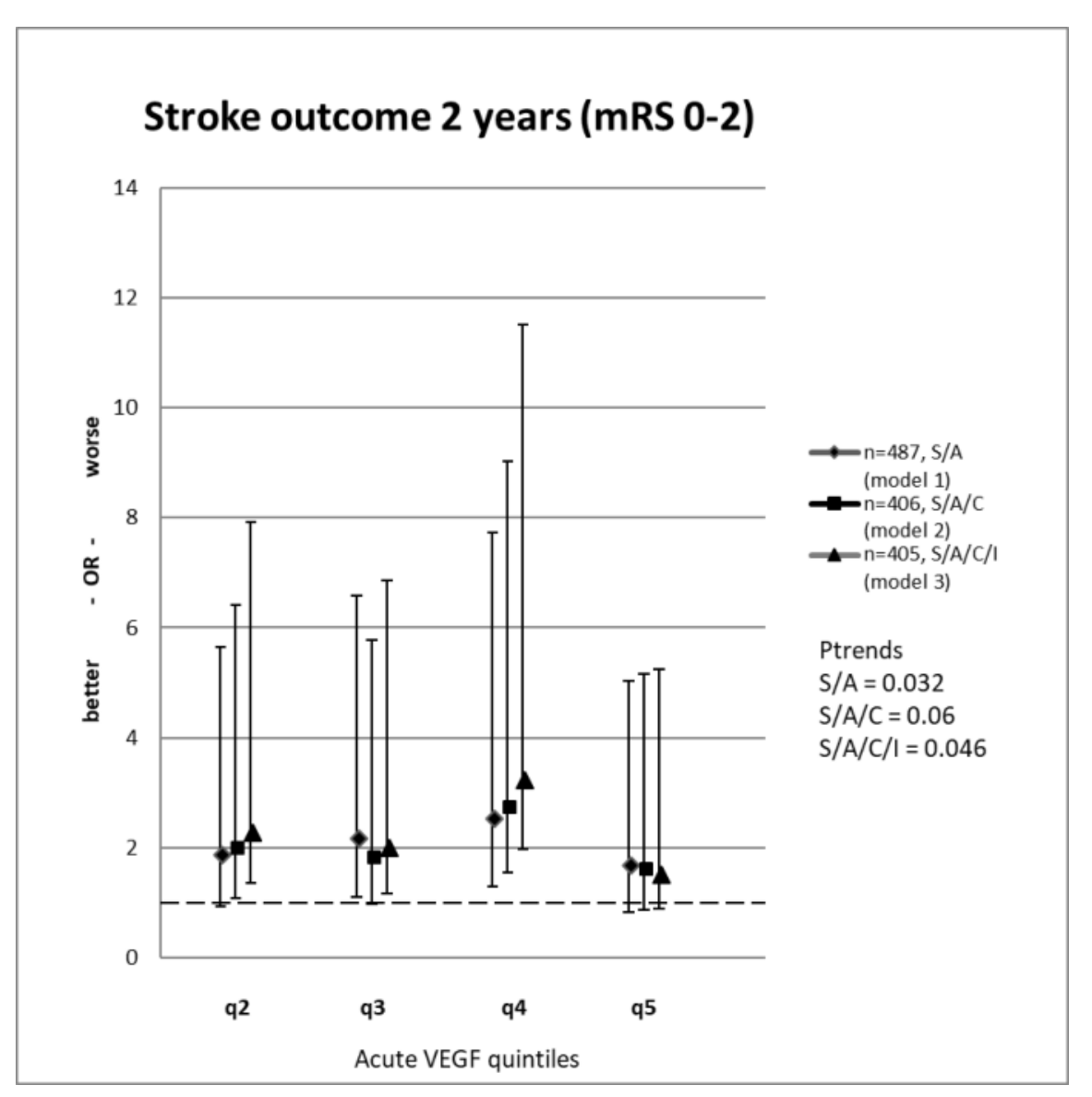

Figure 2. Stroke outcome in relation s-VEGF. Functional outcome 2 years after IS shown by odds ratios (OR) and 95\% confidence intervals $(\mathrm{Cl})$ for associations (binary logistic regression) of unfavorable $\mathrm{mRS}$ over favorable functional outcome in each of the s-VEGF-quintiles relative to s-VEGF-q1 (q1 is reference with $O R=1$, shown as a hatched line). Models 1-3 are shown with successive added adjustments for sex (S), age (A), traditional cardiovascular covariates $(C)$, and initial stroke severity $(\mathrm{I})$ with their respective numbers $(n)$ of complete data. The boxes show the $\mathrm{p}$-values of the overall associations using s-VEGF-quintiles as a continuous variable with the same respective adjustments (ptrends).

\section{Conclusions:}

High s-VEGF is moderately associated with poor longterm outcome, which is not mediated by initial stroke severity. 